RS Open Journal on Innovative Communication Technologies • Issue 5

\title{
A Novel Energy Harvesting Antenna Design for Battery-less loT devices
}

Carlos Eduardo Barrios Salazar ${ }^{1}$

${ }^{1}$ ABU

Published on: Sep 30, 2021

License: Creative Commons Attribution-NonCommercial-NoDerivatives 4.0 International License (CC-BY-NC-ND 4.0). 


\begin{abstract}
Energy harvesting is a process in which small amounts of energy are captured from radiative radio frequencies $(\mathrm{RF})$ that would otherwise be lost as heat, light, sound, or movement. The captured energy allows improving the efficiency of wireless communications, where it enables new technologies such as battery-less devices or power internet of things (IoT) devices without the need for batteries. This paper presents a novel antenna design, called an icosahedron-Sierpiński fractal monopole antenna, where the advantage of such a geometry is in its ability to capture a wide range of frequencies due to its multi-band characteristic and three-dimensional characteristics. The performance of the antenna has been evaluated by numerical simulations and compared with the already existing research. The proposed antenna geometry has been designed using AUTOCAD2020 and simulated on COMOSOL 5.4 to operate at frequencies between $0.84 \mathrm{GHz}, 1 \mathrm{GHz}, 1.6 \mathrm{GHz}, 2.4 \mathrm{GHz}$, and $3 \mathrm{GHz}$.

INDEX TERMS: Energy Harvesting, Fractals, Icosahedron, IoT, Platonic Solids, Radio Frequencies, Sierpiński.
\end{abstract}

\title{
DOWNLOAD ARTICLE PDF
}

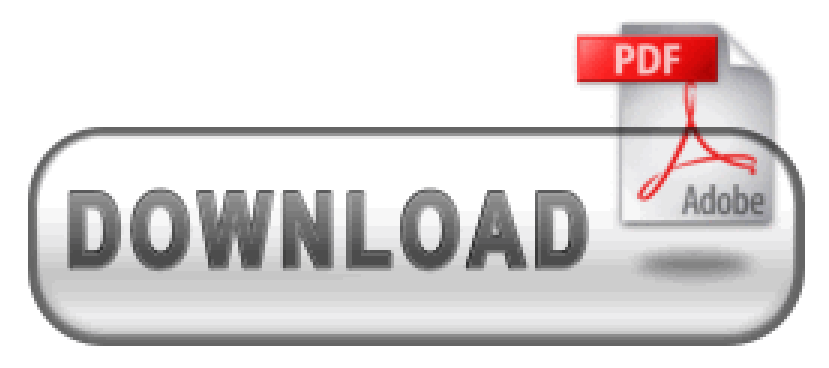

\section{DOWNLOAD SIMULATION CODES}




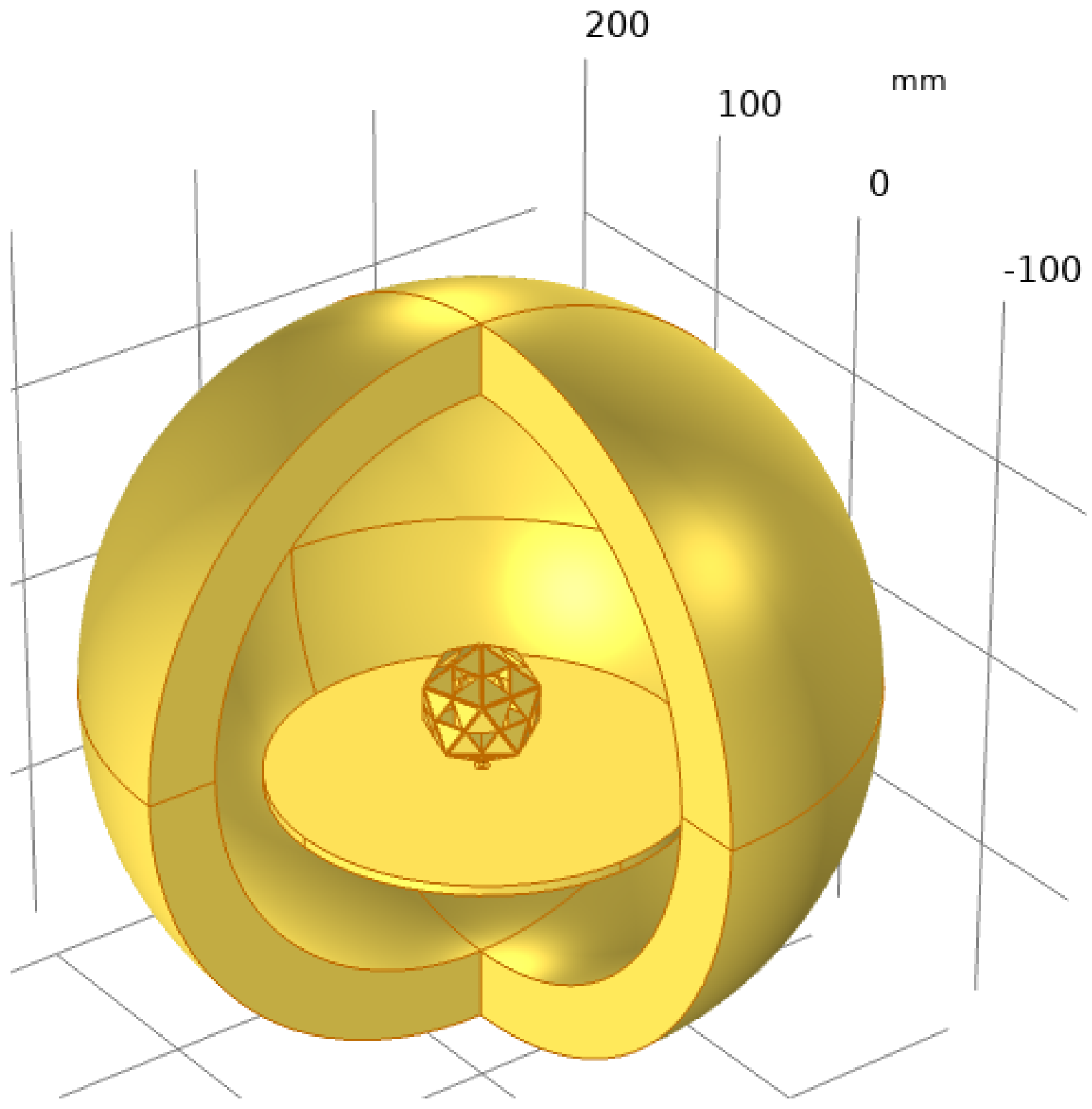

\section{References}

[1] Ewing, J. J. (2018).Electromagnetic Properties of Fractal Antennas(Doctoral dissertation, Wright State University).

[2] Divakaran, S. K., \& Krishna, D. D. (2019). RF energy harvesting systems: An overview and design issues.International Journal of RF and Microwave Computer-Aided Engineering, 29(1), e21633. 
[3] Piñuela, M., Mitcheson, P. D., \& Lucyszyn, S. (2013). Ambient RF energy harvesting in urban and semi-urban environments.IEEE Transactions on microwave theory and techniques,61(7), 2715-2726.

[4] Andrenko, A. S., Lin, X., \& Zeng, M. (2015, Novem $\square$ ber). Outdoor RF spectral survey: A roadmap for ambient RF energy harvesting. In TENCON 2015-2015 IEEE Region 10 Conference(pp. 1-4). IEEE.

[5] Lu, X., Wang, P., Niyato, D., Kim, D. I., \& Han, Z. (2014). Wireless networks with RF energy harvesting: A contemporary survey.IEEE Communications Surveys \& Tutorials, 17(2), 757-789.

[6] S. Porselvi, Sanjay Kumar Suman and L. Bhagyalakshmi "Harvesting RF energy for Mobile Charging” , Australian Journal of Basic and Applied Sciences, June. 2015.

[7] Bakytbekov, A., Nguyen, T. Q., Huynh, C., Salama, K. N., \& Shamim, A. (2018). Fully printed 3D cube-shaped multiband fractal rectenna for ambient RF energy harvesting. Nano Energy, 53, 587-595.

[8] Shi, Y., Jing, J., Fan, Y., Yang, L., Li, Y., \& Wang, M. (2018). A novel compact broadband rectenna for ambient RF energy harvesting.AEU-International Journal of Electronics and Communications,95, 264-270.

[9] Bai, X., Zhang, J. W., \& Xu, L. J. (2017, August). A broadband CPW fractal antenna for RF energy harvesting. In 2017 International Applied Computational Electromagnetics Society Symposium (ACES) (pp. 1-2). IEEE.

[10] Ren, Y. J., \& Chang, K. (2006). 5.8-GHz circularly polarized dual-diode rectenna and rectenna array for microwave power transmission.IEEE Transactions on Microwave Theory and Techniques,54(4), 1495-1502.

[11] Zeng, M., Andrenko, A. S., Tan, H. Z., Lu, C., \& Liu, X. (2016, May). Fractal loop antenna with novel impedance matching for RF energy harvesting. In 2016 Asia-Pacific International Symposium on Electromagnetic Compatibility (APEMC) (Vol. 1, pp. 966968). IEEE.

[12] Andrenko, A. S. (2005, October). Conformal fractal loop antennas for RFID tag applications. In 2005 18th International Conference on Applied Electromagnetics and Communications (pp. 1-6). IEEE. 
[13] Madray, I., Kuemper, R., Martin, J., \& Madani, M. R. (2017, September). Design and COMSOL simulation of Koch snowflake dipole fractal antennas. In 2017 IEEE

International Conference on RFID Technology \& Application (RFID-TA) (pp. 111-115). IEEE. 